\title{
Médiévales
}

Langues, Textes, Histoire

$64 \mid$ printemps 2013

Temporalités de l'Égypte

\section{Les relations entre Italie méridionale, Sicile et Maghreb au Moyen Âge : autour de trois ouvrages récents}

Relationships between Southern Italy, Sicily and the Maghreb in the Middle Ages: about three recent books

\section{Dominique Valérian}

\section{OpenEdition \\ Journals}

Édition électronique

URL : https://journals.openedition.org/medievales/7014

DOI : $10.4000 /$ medievales. 7014

ISSN : $1777-5892$

\section{Éditeur}

Presses universitaires de Vincennes

\section{Édition imprimée}

Date de publication : 1 juillet 2013

Pagination : 173-182

ISBN : 978-2-84292-371-6

ISSN : 0751-2708

\section{Référence électronique}

Dominique Valérian, "Les relations entre Italie méridionale, Sicile et Maghreb au Moyen Âge : autour de trois ouvrages récents », Médiévales [En ligne], 64 I printemps 2013, mis en ligne le 25 septembre 2013, consulté le 23 avril 2022. URL : http://journals.openedition.org/medievales/7014 ; DOI : https:// doi.org/10.4000/medievales.7014 
Médiévales 64,printemps 2013,p. 173-182

Dominique VALÉRIAN

\section{LES RELATIONS ENTRE ITALIE MÉRIDIONALE, SICILE ET MAGHREB AU MOYEN ÂGE: AUTOUR DE TROIS OUVRAGES RÉCENTS}

L'École française de Rome a récemment publié trois ouvrages ${ }^{1}$ qui, sans être centrés sur la question des relations de l'Italie du Sud et de la Sicile avec le Maghreb, permettent chacun de revenir sur un sujet qui est longtemps resté relativement marginal dans l'historiographie, aussi bien italienne que française ou maghrébine. Celle-ci a été longtemps dominée par les recherches sur l'expansion économique et commerciale des cités-États du nord de la péninsule - Gênes ${ }^{2}$, Pise $^{3}$ et Venise ${ }^{4}$ pour l'essentiel. Le Mezzogiorno était le plus souvent relégué dans un passé glorieux qu'incarne la réussite aussi éclatante qu'éphémère d'Amalfi, pionnière - avec Venise - des relations avec l'Islam mais sombrant dans un déclin irrémédiable après les conquêtes normandes. Celles-ci sont tenues pour responsables d'un changement dans la nature des relations entre les ports de Campanie et le monde musulman, les faisant basculer d'un état idyllique de cordialité marchande à un état de conflit permanent teinté d'idéologie de guerre

1. G. L. Borghese, Carlo I d'Angiò e il Mediterraneo. Politica, diplomazia e commercio internazionale prima dei Vespri, Rome, 2008 (Collection de l'École française de Rome, 411); B. Grévin éd., Maghreb-Italie. Des passeurs médiévaux à l'orientalisme moderne (XIII'-milieu $X X^{e}$ siècle), Rome, 2010 (Collection de l'École française de Rome, 439); et A. NEF, Conquérir et gouverner la Sicile islamique aux XI et XII siècles, Rome, 2011 (BEFAR, 346).

2. G. JEHEL, Les Génois en Méditerranée occidentale (fin XI $\mathrm{X}^{\mathrm{A}}$-début XIV siècle). Ébauche d'une stratégie pour un empire, s. 1., 1993.

3. M. Bensaci, Pise et le Maghreb au Moyen Âge, doctorat de troisième cycle, sous la dir. de C.-E. Dufourcq, Université Paris X, 1979.

4. B. Doumerc, Venise et l'émirat hafside de Tunis (1231-1535), Paris, 1999. 
sainte ${ }^{5}$. Seuls émergent ou presque la monumentale thèse d'Henri Bresc ${ }^{6}$, et certains de ses articles, qui éclairent le rôle de la Sicile dans les relations avec le Maghreb oriental, et plus récemment le volume de synthèse de la thèse sur travaux de Philippe Gourdin, malheureusement resté inédit, sur Les Relations politiques et économiques entre l'Italie tyrrhénienne et le Maghreb au XV siècle ${ }^{7}$. Cette marginalité surprend d'autant plus que l'on est là dans une des régions les plus centrales de la Méditerranée, lieu de passage, d'échanges et de conflits par excellence, à la jonction des deux bassins de la Méditerranée et dans une de ces zones où la côte africaine se rapproche considérablement de celle de l'Europe caractéristique qu'elle partage avec le détroit de Gibraltar.

On peut avancer plusieurs raisons à cette relative faiblesse des études sur les relations entre Italie méridionale et Maghreb. La première tient à l'inégalité des sources disponibles, et plus particulièrement des fonds d'archives ${ }^{8}$. L'absence de documents éclairant le commerce avec le Maghreb dans les archives de Campanie ou de Sicile avant une période relativement tardive, aggravée par la perte, en 1943, des originaux des registres angevins de Naples, contraste avec l'abondance, même relative, des documents conservés dans les archives des ports septentrionaux. La deuxième raison est d'ordre historiographique et tient au poids du modèle des «Deux Italies», proposé en 1977 par David Abulafia, et depuis largement accepté 9 . S'inscrivant dans une réflexion plus large et durable sur la périphérisation des $\operatorname{Sud}^{10}$, il montrait un Mezzogiorno privé de toute initiative, notamment sur le plan économique et commercial, par la puissance du capitalisme qui se développe à partir du XII ${ }^{\mathrm{e}}$ siècle dans les ports du nord de la Tyrrhénienne. La région devenait dès lors une simple étape, inerte, sur la route de l'Orient et du Maghreb, alors que l'histoire se faisait, désormais, ailleurs. L'autre ombre dans laquelle s'inscrit cette historiographie est le paradigme des relations - forcément pacifiques car fondées sur le commerce - entre les républiques marchandes et le Maghreb, qui offre une image idéale de ce que pourraient être les relations entre

5. De manière révélatrice le Sud de l'Italie et la Sicile n'apparaissent, dans le livre consacré par G. Jehel aux relations entre l'Italie et le Maghreb, que dans la première partie à travers le récit des relations politiques, marquées par les guerres, alors que la seconde partie, sur la diplomatie et le commerce, ne fait plus guère intervenir que les cités du Nord: G. JeHEL, L'Italie et le Maghreb au Moyen Âge. Conflits et échanges du VIII au $\mathrm{XV}^{e}$ siècle, Paris, 2001.

6. H. BRESC, Un monde méditerranéen. Économie et société en Sicile, 1300-1450, Rome, 1986.

7. Thèse d'État, Université Paris 1, sous la direction de Michel Balard, 2001, volume de synthèse. Il s'y appuie notamment sur les archives de Trapani.

8. D. VALÉRIAn, Les Sources italiennes de l'histoire du Maghreb médiéval. Inventaire critique, Paris, 2006.

9. D. Abulafia, The Two Italies. Economic Relations between the Norman Kingdom of Sicily and the Northern Communes, Cambridge, 1977.

10. M. T. Pérez Picazo, G. Lemeunier éd., Desigualdad y dependencia. La periferización del Mediterráneo occidental (s.XII-XIX), Murcie, 1986. 
l'Europe et le Maghreb, que ce soit à l'époque coloniale ${ }^{11}$ ou post-coloniale ${ }^{12}$. Dans le cadre de ce discours irénique sur la Méditerranée, les relations complexes entre le Maghreb et le Sud péninsulaire ou la Sicile, marquées notamment par des conflits permanents autour d'enjeux de contrôle territorial ou des routes maritimes, introduisaient une note discordante ${ }^{13}$. Enfin il faut souligner une dernière raison, qui concerne surtout les relations culturelles, au cœur du projet de Benoît Grévin: le Maghreb est souvent présenté comme une région marginale dans le mouvement de traductions et de transferts culturels entre l'Islam et le monde latin, qui se serait surtout déployé en Péninsule ibérique, en Sicile ou au Proche-Orient - ce que B. Grévin qualifie de «complexe oriental». Cette vision rejoint d'ailleurs celle, plus globale, d'un Maghreb inerte et réceptacle passif d'influences extérieures ou simple voie de passage de flux qui trouvent leur logique ailleurs, qui a été longtemps une des thèses majeures de l'historiographie coloniale et au-delà. C'est cette double marginalité - du Maghreb par rapport à l'Orient et à al-Andalus, et du Mezzogiorno par rapport à l'Italie du Nord - que ces trois ouvrages permettent de relativiser en réinterrogeant les relations entre ces deux espaces et en les replaçant au centre de la réflexion sur la Méditerranée.

Le livre d'Annliese Nef, produit d'une thèse soutenue en 2001 à l'université de Nanterre et largement remaniée ${ }^{14}$, porte sur l'époque normande en Sicile et sur la place des populations arabo-musulmanes majoritaires dans la construction originale de la monarchie normande. Celui de Gian Luca Borghese reprend la riche documentation des registres angevins reconstitués pour brosser un tableau de la politique méditerranéenne des Angevins avant les Vêpres siciliennes,

11. B. GRÉvin, «Le quatuor de Berbérie: note sur la place de l'Italie dans la construction d'un savoir maghrébin par les réseaux académiques du premier XIXe siècle (ca 1815-ca 1870)», dans ID. éd., Maghreb-Italie..., p. 267-284; D. VALÉRIAN, «Lectures italiennes de l'expansion latine dans le Maghreb médiéval (première moitié du xxe siècle)», dans B. GRÉvin éd., Maghreb-Italie..., p. 343356.

12. S. Mourlane, "Un pont entre l'Orient et l'Occident": paradigme médiéval, médiation culturelle et politique arabe de l'Italie des années 1950 », dans B. Grévin éd., Maghreb - Italie..., p. 435-446.

13. Certaines périodes sont marquées par des relations diplomatiques particulièrement tendues, notamment dans la seconde moitié du Xve siècle avec les rois d'Aragon maîtres de la Sicile et du royaume de Naples. D. VALÉRIAN, «Les relations entre l'Italie et le Maghreb dans la seconde moitié du Xv $v^{\mathrm{e}}$ siècle. Les conditions d'un nouvel essor», dans B. GRÉvin éd., Maghreb-Italie..., p. 89. Il faudrait cependant nuancer ce que j' affirmais alors, que les relations commerciales étaient limitées avec le Mezzogiorno à cette époque : le témoignage des archives de Trapani, comme l'importance de la traite des esclaves des Monts de Barca par les marchands catalans à partir de leurs bases siciliennes, montrent que la dégradation des relations politiques n'empêchait pas les échanges commerciaux, parfois interlopes.

14. A. NeF, L'Élément islamique dans la Sicile normande. Identités culturelles et construction d'une nouvelle royauté (XI $-X I I^{e}$ siècle), thèse de l'Université de Nanterre, sous la direction d'Henri Bresc, 2001. 
et consacre un chapitre, en partie repris en français dans l'ouvrage dirigé par B. Grévin, aux relations avec le Maghreb. Ce dernier livre est le produit de trois journées d'études organisées à Florence et Rome en 2006 et 2007 et aborde la question des transferts culturels et plus largement de la connaissance que l'on avait du Maghreb en Italie, de la fin du Moyen Âge au milieu du Xx $x^{e}$ siècle, avec donc une large part de réflexion historiographique mais aussi des contributions sur les modalités des relations (politiques ou économiques) entre les deux régions. L'Italie méridionale n'avait donc pas vocation à être davantage traitée que le Nord, mais elle y trouve pourtant une place plus importante et significative.

Dans ces trois ouvrages la singularité de la relation entre le Mezzogiorno et le Maghreb apparaît d'abord à travers des modèles originaux de relations diplomatiques entre puissances chrétiennes et musulmanes, très différentes en tout cas de celles de républiques du Nord. Mais elle est liée aussi à la configuration particulière des réseaux de relations entre les deux régions, marquée à la fois par des relations de proximité de part et d'autre du canal de Sicile et par une intégration forte dans des réseaux à longue distance entre les deux bassins de la Méditerranée.

À la différence des républiques du Nord de l'Italie, intéressées avant tout à garantir la sécurité de leurs navires et de leurs marchands, et à obtenir les meilleures conditions possibles de séjour et de commerce au Maghreb, les monarchies qui se succèdent dans le Sud entretiennent avec les puissances maghrébines - essentiellement l'Ifrîqiya, à l'Est - des relations plus complexes où les intérêts commerciaux ne sont pas absents, mais se mêlent à des considérations de politique régionale, de contrôle de l'espace maritime autour du canal de Sicile, voire de conquêtes territoriales. Comme le remarque justement A. Nef, la position de la Sicile, face à une Ifrîqiya en plein bouleversement politique, laisse peu de place à la neutralité des souverains normands. Par ailleurs, les ambitions méditerranéennes de ces derniers, comme, par la suite, des Souabes et des Angevins et après eux des rois d'Aragon, contribuent un peu plus encore à une politique d'intervention directe dans les affaires maghrébines, qui peut prendre des formes militaires parfois, plus souvent politiques. En ce sens cette politique s'apparente sur bien des points avec celle des monarchies chrétiennes de la Péninsule ibérique, mais avec un arrière-plan idéologique et surtout géostratégique différent. En apparence en effet les points communs sont nombreux et placent ces politiques sous le signe de l'expansion territoriale chrétienne et des relations de frontière. On y retrouve des conquêtes territoriales, à commencer par celle de la Sicile elle-même, dont on peut voir un prolongement avec la politique de Roger II en Ifrîqiya, avec des mécanismes qui ne sont pas sans rappeler les interventions des princes ibériques dans les émirats de taïfas divisés. La faiblesse des Zirides et les conflits avec les Hammadides, les Almoravides ou des petits seigneurs locaux facilitent la conquête autant qu'ils donnent une 
légitimité à l'intervention normande. Cette domination, limitée aux ports et aux îles, qui permettent le contrôle des routes maritimes, ne cherche cependant pas à s'implanter en profondeur, dans un pays il est vrai compliqué à contrôler et, en dépit de l'instauration d'une administration qui emprunte beaucoup à l'expérience sicilienne, les Normands ne peuvent se maintenir au-delà de la conquête almohade de 1159. Il est donc difficile de parler alors de front de conquête, comme on pourrait le voir en Péninsule ibérique, mais plutôt d'une stratégie de domination fortement articulée autour du contrôle de la mer et des routes maritimes. On la retrouve plus tard, avec la couronne d'Aragon et une politique ouvertement dirigée vers le contrôle des îles: la Sicile et la Sardaigne bien sûr, mais aussi, sous des formes différentes, les îles d'Ifrîqiya de Djerba et des Kerkennah. L'exigence du tribut est un autre trait de la politique des souverains de Sicile et d'Italie du Sud vis-à-vis des musulmans d'Ifrîqiya que l'on peut comparer à la situation ibérique, avec le système des parias. Le tribut, imposé à Tunis par Frédéric II et maintenu par Manfred, cesse d'être versé à Charles d'Anjou qui y voit le signe d'une menace ifrîqiyenne pour la sécurité de la Sicile, ce qui a pu être une des motivations de la croisade de 1270 - qui se solde d'ailleurs par son rétablissement et le paiement des arriérés. Il est ensuite régulièrement au cœur des relations diplomatiques compliquées entre Tunis et les rois d'Aragon, qui en revendiquent l'héritage au titre de leur couronne de Sicile. Il y a là une volonté de se voir reconnaître par les sultans hafsides une forme de supériorité politique, ainsi qu'une source appréciable de revenus versés en or. Mais la comparaison avec la Péninsule ibérique s'arrête là, car ce tribut ne débouche jamais sur un mécanisme de conquête territoriale durable. Enfin on pourrait rapprocher les projets idéologiques ibériques et normands de l'émergence précoce d'un esprit de guerre sainte, parfois présenté comme un prélude à la croisade orientale. Mais A. Nef montre bien que, en dépit d'une «religiosité diffuse», notamment dans certains textes, et du soutien parfois de la papauté, ce qui domine est avant tout le «caractère pragmatique, sur le plan politique et militaire, de la conquête normande $»^{15}$ - pragmatisme que l'on retrouve ensuite dans la politique des Normands à l'égard des groupes arabo-musulmans, au cœur de la thèse d'A. Nef. Les motivations de Charles d'Anjou dans la croisade de 1270, souvent opposées à celles de son frère Louis IX, montrent à cet égard une réelle continuité. Ce pragmatisme ne vaut d'ailleurs pas que pour les souverains, comme le montre la politique d'exportation de céréales en direction du Maghreb étudiée par G. L. Borghese: l'Ifrîqiya n'est en effet pas incluse dans les destinations pour lesquelles la papauté impose un embargo, comme l'Égypte mamelouke. Cette exception, que l'on peut rapprocher de l'autorisation accordée aux Majorquins 
en 1241 de commercer avec le Maghreb et justifiée par l'importance vitale de ce commerce pour l'île, obéit surtout à la nécessité d'écouler la production céréalière du royaume angevin. Là encore, la continuité avec les périodes précédentes est évidente, comme le montre G. Mandalà à propos de la politique de Frédéric II, qui visait à la fois à tirer un profit maximal de la vente des grains (en l'occurrence au Maghreb ou en al-Andalus où sévissait alors une famine) et à se fournir en or nécessaire à la frappe des augustales et au financement de la politique de l'empereur ${ }^{16}$. Mais ces exportations contrôlées visent aussi à faire des céréales une arme diplomatique au service de la politique de Charles en Méditerranée, et plus particulièrement en direction du Maghreb. Les relations diplomatiques et militaires doivent donc être lues moins en termes d'idéologie ou de légitimation d'un pouvoir par la lutte contre l'infidèle, assez peu visibles dans les politiques mises en œuvre comme dans les discours, que dans le cadre des stratégies à la fois régionales et méditerranéennes des souverains normands, souabes, angevins puis aragonais.

L'échelle régionale, qui permet d'étudier les relations de proximité de part et d'autre du canal de Sicile et qui est souvent négligée au profit des mouvements de plus grande ampleur, est dans ces ouvrages au contraire centrale, en particulier pour la Sicile. G. L. Borghese montre bien l'importance de courants d'échanges marchands entre les domaines angevins et le Maghreb, qui débordent de la question du commerce frumentaire et impliquent des marchands d'Italie méridionale, notamment de Gaète et Amalfi. Cela nous est confirmé, au siècle suivant, par les manuels de marchands comme celui de Pegolotti, qui soulignent l'importance de ce courant d'échanges, et plus tard par les documents des notaires de Trapani. Au-delà des aspects commerciaux ou politiques, cette échelle régionale d'analyse permet de mettre en évidence des formes de proximité qui font de la Sicile un relais privilégié pour les transferts culturels. B. Grévin propose même de rouvrir le «débat sur l'inclusion de la Sicile dans les définitions du Maghreb»:

S'il y aurait abus à faire rentrer systématiquement l'histoire de la grande île dans celle du Maghreb, il est sûr que la Sicile islamique, puis islamo-normande, enfin la Sicile arabophone résiduelle des communautés juives des deux derniers siècles du Moyen Âge entretenait des liens linguistiques et culturels particulièrement étroits avec le Maghreb (non exclusifs, d'ailleurs, de liens importants avec d'autres régions du monde islamique $)^{17}$.

16. G. Mandalà, «La migration des juifs du Garbum en Sicile (1239)», dans B. Grévin éd., Maghreb-Italie..., p. 37.

17. B. GRÉvin, «Maghreb-Italie, des passeurs médiévaux à l'orientalisme moderne. Apories scientifiques et stratégie de contournement», dans Id., Maghreb-Italie..., p. 14. C'est dans ce débat 
À l'inverse, le Maghreb est surtout étudié et exploité par Michele Amari dans une optique de construction de son histoire sicilienne et italienne. La langue arabe joue à cet égard un rôle majeur, et elle occupe une place centrale, dans la thèse d'A. Nef, comme un des outils mobilisés par les souverains normands pour leur encadrement des populations arabo-musulmanes, et, dans l'ouvrage de B. Grévin, comme un des vecteurs sur le temps long de transmission d'un savoir islamique vers l'Italie. Le mouvement de traductions y est cependant limité, et on n'en a plus de trace après celle du Kitāb al-ḥâwwī d'al-Rāzī, que Charles d'Anjou reçoit en cadeau du sultan hafside et qu'il fait traduire dans les années 1270. L'expulsion des populations musulmanes de Sicile est par ailleurs plus précoce qu'en Péninsule ibérique, avec leur déportation à Lucera par Frédéric II. Pourtant, B. Grévin souligne la «permanence d'une arabité sicilienne, liée au Maghreb, au sens d'Occident arabophone ${ }^{18}$. Celle-ci passe, après l'expulsion des musulmans, par les communautés juives qui assurent une fonction de passeurs, principalement culturels. Leur rôle d'intermédiaires dans le commerce à longue distance, qui avait été majeur en Sicile à l'époque de la Geniza et dans le cadre d'un espace de relations fatimide ${ }^{19}$, ne semble plus être considérable, et on voit bien davantage les marchands des grandes cités du Nord de l'Italie ou, après les Vêpres, de la couronne d'Aragon. Cela contraste avec leur place, soulignée par Ingrid Houssaye, dans les échanges entre Majorque (où réside également une forte communauté juive) et le Maghreb, telle que l'éclairent les documents de la compagnie Datini de Prato $^{20}$. En revanche, ils jouent un rôle majeur, déjà montré par Henri Bresc ${ }^{21}$, dans le maintien de la pratique de l'arabe dans l'île. L'article de Giuseppe Mandalà sur l'immigration des juifs du Garbum (identifié à juste titre avec le Maghreb occidental) montre en effet que non seulement la Sicile conserve sa population juive, mais qu'elle est aussi un pôle d'attraction et d'immigration pour les communautés en difficulté au Maghreb, signe supplémentaire des liens étroits qui unissent alors la Sicile à l'autre rive. Ces juifs sont en tout cas porteurs d'une arabophonie qui perdure en Sicile jusqu'à l'expulsion de 1492, avec des parlers proches de ceux du Maghreb. C'est ce

que se positionne A. Nef par le titre, quelque peu provocateur, de son livre qui fait aller la Sicile islamique jusqu' au $\mathrm{XII}^{\mathrm{e}}$ siècle.

18. B. GRÉvin, «Connaissance et enseignement de l'arabe», dans ID. éd., Maghreb-Italie..., p. 106.

19. A. NeF, «La Sicile dans la documentation de la Geniza cairote (fin $\mathrm{X}^{\mathrm{e}}$-XIII ${ }^{\mathrm{e}}$ siècle): les réseaux attestés et leur nature», dans D. Coulon, C. PiCARD et D. VALÉRIAN éd., Espaces et réseaux en Méditerranée (VI'-XVI siècle), vol. I: La Configuration des réseaux, Paris, 2007, p. 273-291.

20. I. Houssaye-Michienzi, «De Prato au Maghreb: le réseau commercial de la compagnie Datini, fin XIV"edébut XV siècle», dans B. GréVIN éd., Maghreb-Italie..., p. 67-84.

21. H. BRESC, Arabes de langue, juifs de religion. L'évolution du judaïsme sicilien dans l'environnement latin, $\mathrm{XII}^{e}$-XV' siècles, Paris, 2001. 
dont témoigne en particulier le «Coran de Mithridate» étudié par B. Grévin: ce manuscrit singulier, copié au début du XV siècle, peut-être en Sicile ou en tout cas sur un papier au filigrane palermitain, est écrit en langue arabe et alphabet hébreu, dans ce judéo-arabe si courant dans les documents de la Geniza et pratiqué par les communautés juives autour de la Méditerranée. C'est ce manuscrit qui, dans les années 1470-1480, dans le contexte de l'humanisme de la cour pontificale, est annoté - en latin, hébreu et arabe - par Guglielmo Raimondo Moncada. Ce juif sicilien converti, qui se fait aussi appeler Flavius Mithridate, est né dans une famille de lettrés et a été formé à l'hébreu et l'araméen, mais aussi à l'arabe dans sa version vernaculaire sicilienne, proche des parlers du Maghreb hafside avec lequel les contacts restaient alors étroits. Très lié aux milieux humanistes - il est le maître d'arabe de Pic de la Mirandole - il est un de ces passeurs d'un savoir livresque arabe venant du Maghreb et ayant transité par la Sicile, vers les milieux savants curieux d'une ouverture vers les langues sémitiques.

Pour autant faut-il déduire de cet exemple, et de quelques autres comme celui du Siennois Beltramo Mignanelli près d'un siècle plus tôt, qu'il y a un flux notable et continu de savoirs entre le Maghreb et l'Italie au cours du Moyen Âge? B. Grévin montre son optimisme en admettant que «le lecteur devra toutefois se contenter des prémices de ces découvertes, en exerçant sa patience ou son imagination pour replacer dans ce continuum d'histoire économique la possibilité de ces échanges culturels qu'on lui fera toucher du doigt à deux ou trois reprises», mais en "prenant le pari que dans quelques années, d'autres aspects de cette histoire italo-maghrébine auront émergé des brumes du passé » ${ }^{22}$. Sans considérer nécessairement Moncada ou Mignanelli comme des exceptions isolées, dues seulement à des parcours et des personnalités hors du commun, il faut aussi mettre ces expériences en relations avec des contextes particuliers qui génèrent des attentes fortes en direction d'un savoir arabe: de nature religieuse pour les besoins de la polémique et de la mission, politique (dans le cas de la traduction d'al-Rāzī pour Charles d'Anjou) ou plus savante dans le cas des cercles humanistes. Par ailleurs, il faut le souligner, si les échanges marchands avec les ports maghrébins permettent l'apprentissage de l'arabe par les marchands latins ${ }^{23}$, les rares exemples de transferts d'un savoir livresque passent par l'intermédiaire des milieux savants juifs de Sicile, et non par des relations intellectuelles directes entre l'Italie et le Maghreb - à l'exception notable de Fibonacci, mais qui est presque le fruit du hasard.

22. B. GRÉvin éd., Maghreb-Italie..., p. 7.

23. C'est le cas de Beltramo Mignanelli, et avant lui du Pisan Leonardo Fibonacci, qui apprit l'arabe en même temps que les mathématiques à Bougie, où il accompagnait son père, scriba de la nation pisane. La pratique de l'arabe, ou du moins de rudiments d'arabe, est bien attestée parmi les marchands installés de manière durable dans les ports musulmans (cf. D. VALÉRIAN, «Les relations entre l'Italie et le Maghreb...», p. 100-102). 
L'exemple de Mignanelli, qui parfait sa connaissance de l'arabe en Orient après en avoir acquis les bases grâce à son activité de marchand à Tunis, montre que, dans le domaine des transferts culturels comme des échanges économiques, les relations à l'échelle régionale interagissent avec des réseaux plus vastes, qui couvrent toute la Méditerranée. C'est bien cet horizon méditerranéen qui est celui des souverains dominant la région, qu'ils aient leur capitale à Palerme, Naples ou, plus tard, à Barcelone, qui éclaire les relations avec le Maghreb. Dès lors se pose aussi la question de la place de cet espace du Mezzogiorno dans les stratégies politiques et commerciales mises en œuvre: est-ce un simple relais, un point d'appui pour atteindre d'autres marchés ou territoires, ou joue-t-il un rôle moteur et structurant dans ces réseaux méditerranéens? Politiquement, mais aussi commercialement et dans l'imaginaire, l'Orient exerce une attraction forte, qui bien souvent conditionne les ambitions et les stratégies. Ainsi, pour les Normands, l'intervention en Ifrîqiya « autorise également la Sicile à développer des ambitions de nature impériale sur le même plan que d'autres ensembles politiques, à justifier le refus de la croisade orientale et à traiter sur un pied, sinon d'égalité du moins de respect mutuel, avec les Fatimides ${ }^{24}$. Dans le même temps les Fatimides leur laissent les mains libres en Ifrîqiya car ils y voient un facteur de stabilité, à condition que leur pouvoir suive les règles islamiques ${ }^{25}$. La politique angevine, que G. L. Borghese étudie précisément dans sa dimension méditerranéenne, permet aussi de voir combien les relations avec le Maghreb sont liées à des préoccupations orientales, que ce soient les efforts de paix de Charles en direction du sultan mamelouk d'Égypte Baybars ou des ambitions à Constantinople ou Jérusalem: alors que, pour le dominicain Geoffroy de Beaulieu, l'attaque contre Tunis devait servir de point d'appui pour une croisade contre l'Égypte, Charles y voyait à l'inverse un moyen d'éviter une attaque croisée contre son allié égyptien. Sur le plan commercial, la possession de la Sicile permet sinon d'imposer un monopole de la navigation entre les deux bassins de la Méditerranée, du moins d'offrir des conditions plus favorables et un point d'appui stratégique. C'est ce dont profitent notamment les marchands catalans pour développer leurs affaires dans le Maghreb oriental, pendant longtemps domaine réservé ou presque des républiques italiennes ${ }^{26}$, mais aussi dans le bassin oriental de la Méditerranée - alors que dans le même temps la possession d'une autre grande île, celle de Majorque, permet aux marchands de la couronne, et plus particulièrement aux

24. A. NeF, Conquérir et gouverner..., p. 597.

25. Ibid.

26. Ils s'imposent notamment au $\mathrm{XV}^{\mathrm{e}}$ siècle, avec les Vénitiens, comme les principaux acteurs du commerce des esclaves noirs expédiés depuis les plages et ports des Monts de Barca (Cyrénaïque) et de Tripolitaine, et ensuite renvoyés depuis la Sicile vers les marchés de la Péninsule ibérique. 
Majorquins, d'imposer un quasi-monopole sur le commerce avec le Maghreb occidental et central ${ }^{27}$.

Les relations entre le Mezzogiorno et le Maghreb présentent donc bien des caractères différents de celles entretenues à la même époque par les républiques maritimes du Nord. Pour autant il n'est pas possible de résumer cette différence à une approche de ces relations qui serait plus politique qu'économique, ou plus violente et marquée par des confrontations idéologiques. Tous ces travaux montrent l'importance de cette région dans les relations complexes qui se nouent au Moyen Âge en Méditerranée, à la fois parce que sa position centrale la place au cœur des compétitions entre puissances, mais aussi parce qu'elle résume à elle seule toute la diversité des relations entre les deux rives, puisqu'on y trouve à la fois, et parfois simultanément, des éléments de conflits (armés, diplomatiques ou commerciaux), des échanges économiques importants, mais aussi des phénomènes de transferts culturels. La proximité des côtes rend plus nécessaire encore que pour d'autres régions une analyse à une double échelle régionale et méditerranéenne. La politique des puissances chrétiennes de la région (Normands, Souabes, Angevins, rois d'Aragon) se comprend en effet en grande partie par la place stratégique de la région dans des réseaux vastes qui permettent d'envisager des politiques de domination de la Méditerranée. Pour autant l'échelle régionale n'est pas moins pertinente, et la région ne peut pas être seulement définie comme une périphérie ou une escale stratégique par rapport à des pôles plus éloignés. L'importance de l'épisode islamique de la Sicile en particulier, et de ses conséquences bien au-delà de la conquête normande, joue sur les relations étroites de part et d'autre du canal de Sicile, qu'incarnent à la fois les échanges commerciaux et le maintien, jusqu'à la fin du $\mathrm{Xv}^{\mathrm{e}}$ siècle, d'une pratique de l'arabe maghrébin par les populations juives. Le Mezzogiorno, et plus encore la Sicile pour laquelle joue en plus l'élément insulaire, présente donc une grande diversité de types de relations avec le Maghreb ${ }^{28}$ : relations de frontière, marquées par la proximité des côtes et la porosité des limites, contacts par l'intermédiaire de populations culturellement à cheval sur les deux espaces (arabo-musulmans, puis juifs), enfin insertion dans des réseaux de relations à différentes échelles, qui peuvent aller jusqu'à mettre en jeu les deux extrémités de la Méditerranée, et aussi bien le Sud que le Nord de la Péninsule italienne.

Dominique VALÉRIAN - Université Lumière Lyon 2, UMR 5648 CIHAM - ISH - 14 av. Berthelot - 69363 Lyon Cédex 07.

27. I. Houssaye-Michienzi, «De Prato au Maghreb...».

28. On pourrait le comparer au détroit de Gibraltar, qui fait l'objet actuellement d'un projet de recherche ANR (DETROIT) et présente beaucoup de similitudes. 